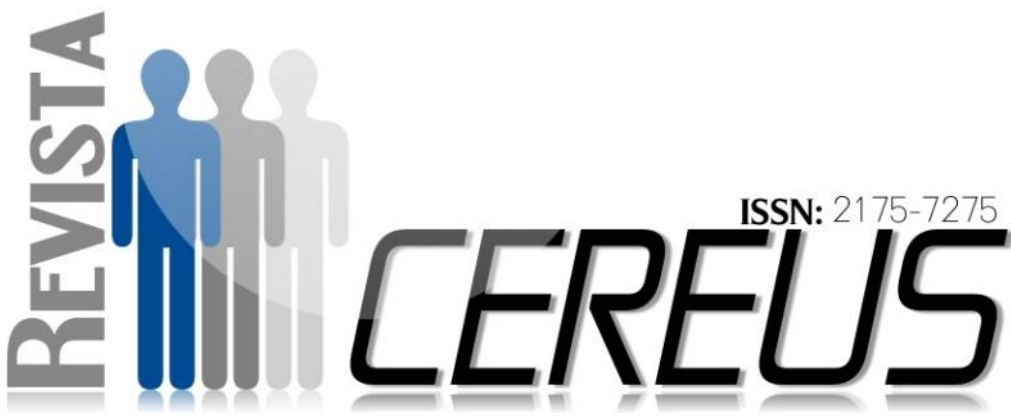

\section{ENFOQUE CTS: CONTRIBUIÇÕES PARA ANÁLISE DO PLANO DE CIÊNCIA, TECNOLOGIA E INOVAÇÃO PARA DESENVOLVIMENTO SUSTENTÁVEL DO NORDESTE BRASILEIRO}

\author{
ALBUQUERQUE, Andreza Dantas ${ }^{1}$ \\ SANTOS, Elis Regina Alves dos ${ }^{2}$; \\ SOUSA, Cidoval Morais de ${ }^{3}$.
}

\section{RESUMO}

A proposta deste paper é discutir, à luz dos pressupostos CTS, o Plano de Ciência, Tecnologia e Inovação para o Desenvolvimento Sustentável do Nordeste Brasileiro (PCTI/NE). O Plano, parte de três constatações: que o papel da CT\&I é fundamental para manutenção das transformações em curso no Nordeste, que mesmo avançando o Nordeste acumula déficits acentuados em várias frentes, e que 0 deslanche técnico-científico pressiona segmentos de maior densidade tecnológica, promovendo intercâmbio com outras regiões do País. Busca-se, assim, uma análise do $\mathrm{PCTI} / \mathrm{NE}$, focada nas seguintes questões: a concepção de CT\&I expressa (ou nas entrelinhas) do documento; a relação CT\&I

\footnotetext{
${ }^{1}$ Doutoranda do Programa de Pós-Graduação em Ciência, Tecnologia e Sociedade da Universidade Federal de São Carlos (PPGCTS/UFSCAR)..

${ }^{2}$ Doutora em Ciência, Tecnologia e Sociedade pela Universidade Federal de São Carlos

3 Professor do PPGDR/ UEPB e colaborador do PPGCTS/UFSCar. cidoval@gmail.com
}

v. 9, n. 3, set/dez. 2017 UnirG, Gurupi, TO, Brasil 
- Desenvolvimento Regional; a natureza das políticas propostas, seu alcance e diálogo com as demandas críticas para o desenvolvimento regional; e como as ações de CT\&I propostas interagem com as estratégias de desenvolvimento para a região. Tal análise será documental, orientada pelos estudos de Renato Dagnino, Hebe Vessuri, Pablo Kreimer, dentre outros, que se articulam no Pensamento Latinoamericano em Ciência, Tecnologia e Sociedade (PLACTS). Compreende-se que o Plano representa uma contribuição importante para o avanço da articulação federativa e o desenvolvimento de projetos, programas e demais iniciativas de CT\&I no Nordeste. Entretanto, só ganhará sentido prático na medida em que encontre expressão concreta na política de CT\&I dos Estados da região e do País, e, principalmente, dialogue com as demandas dos diferentes "Nordestes".

Palavras chave: Plano de Ciência, Tecnologia e Inovação para o Desenvolvimento Sustentável do Nordeste Brasileiro (PCTI/NE). Ciência, Tecnologia e Sociedade (CTS). Desenvolvimento regional.

\section{CTS APPROACH: CONTRIBUTIONS FOR ANALYSIS OF THE SCIENCE, TECHNOLOGY AND INNOVATION PLAN FOR THE SUSTAINABLE DEVELOPMENT OF THE BRAZILIAN NORTHEAST (PCTI / NE)}

\section{ABSTRACT}

The proposal of this paper is to discuss, in the light of the CTS assumptions, the Science, Technology and Innovation Plan for the Sustainable Development of the Brazilian Northeast (PCTI / NE). The Plan, part of at least three findings: a) the maintenance of the transformation process underway in the Northeast reserves a key role for the CT \& I; b) the Northeast has advanced a lot in recent years, but still accumulates accentuated deficits on several fronts (the country has the highest inequality index); and c) the departure of the technical- 
scientific base forces the incursion into segments of greater technological density and promotes greater exchange with existing competences in other regions of the country. This work seeks an analysis of the PCTI / NE, focusing on the following issues: the design of CT \& I expressed (or between the lines) of the document; the CT \& I - Regional Development relationship; the nature of the proposed policies, their scope and dialogue with the demands critical to the development of the region; and also how the CT \& I actions proposed interact with the other development strategies for the region. This analysis will be guided by the studies of Renato Dagnino, Hebe Vessuri, Pablo Kreimer, among others, which are articulated in the Latin American Thinking in Science, Technology and Society (PLACTS). It is understood that the Plan represents an important contribution for the advancement of the federative articulation and the development of projects, programs and other CT \& I initiatives in the Northeast. However, it will only gain practical meaning insofar as it finds concrete expression in the ST \& I policy of the States of the region and of the Country, and, above all, dialogue with the demands of the different "Northeast.".

Keywords: Science, Technology and Innovation Plan for the Sustainable Development of the Brazilian Northeast (PCTI / NE). Science, Technology and Society (CTS). Regional development 


\section{INTRODUÇÃO}

Avanços recentes na Política de Ciência, Tecnologia e Inovação (PCT\&I) Nacional demonstram que a pactuação entre a União e os Estados tem ampliado o escopo e efetivação das iniciativas em Ciência, Tecnologia e Inovação (CT\&l) no país, consolidando um Sistema Nacional de Ciência, Tecnologia e Inovação (SNCTI) estruturado e abrangente. A construção do documento Estratégia Nacional de Ciência, Tecnologia e Inovação 2016-2019, pelo então Ministério da Ciência, Tecnologia e Inovação (MCTI), dá continuidade, aprimoramento e atualização a documentos similares anteriores, como a Estratégia Nacional de CT\&I 20122015 (ENCTI) e o Plano de Ação em Ciência, Tecnologia e Inovação 20072010 (PACTI) (BRASIL, 2016). As tendências que prevalecem neste sentido giram em torno da descentralização de programas, articulação para o estabelecimento de patamares mínimos de contrapartida aos repasses federais e desenhos cooperativos de iniciativas de fôlego no âmbito da CT\&l (CENTRO DE GESTÃO E ESTUDOS ESTRATÉGICOS, 2014).
O desenvolvimento do Plano de Ciência, Tecnologia e Inovação para o Desenvolvimento Sustentável do Nordeste Brasileiro (PCTI/NE) decorre deste contexto. Encomendado pelos secretários de CT\&I da região e presidentes das Fundações de Amparo à Pesquisa (FAP) ao Centro de Gestão e Estudos Estratégicos (CGEE) e encampado pelo MCTI, o plano procura levar em consideração as especificidades e desafios da região, pensando a CT\&l no bojo do desenvolvimento regional sustentável, e em consonância com o ENCTI, reforçando as diretrizes necessárias para a consolidação de uma CT\&I efetiva (CENTRO DE GESTÃO $E$ ESTUDOS ESTRATÉGICOS, 2014).

Assim, a proposta deste paper é discutir o PCTI/NE à luz do Enfoques CTS (Ciência, Tecnologia e Sociedade). Por ser um campo de estudo interdisciplinar, nos permite dialogar com outros campos, como o próprio Desenvolvimento Regional, com o intuito de engrandecer a discussão que aqui se propõe sobre as estratégias que guiarão 0 desenvolvimento tecnológico da região Nordeste nos próximos vinte anos, 
levando em consideração que os Estudos Sociais da Ciência e Tecnologia defendem uma Política de Ciência e Tecnologia (PCT) alinhada para atender as necessidades da maioria da população, ou seja, cada vez mais democrática, construída para e pela sociedade e capaz de promover o desenvolvimento socioeconômico da região.

A análise buscar problematizar: a concepção de CT\&I expressa (ou nas entrelinhas) do documento; a relação CT\&I - Desenvolvimento Regional; a natureza das políticas propostas, seu alcance e diálogo com as demandas críticas para o desenvolvimento da região; e, ainda, como as ações de CT\&I propostas interagem com as demais estratégias de desenvolvimento para a região.

Metodologicamente, o artigo é uma investigação documental, a partir da analise do PCTI/NE, fundamentado em uma revisão de literatura entre os principais pesquisadores do Pensamento Latino-americano em Ciência, Tecnologia e Sociedade, entre eles Renato Dagnino, Hebe Vessuri e Pablo Kreimer.

\section{O CAMPO CTS E SUA CONTRIBUIÇÃO TEÓRICO-CONCEITUAL SOBRE CIÊNCIA E TECNOLOGIA}

O campo de estudos Ciência, Tecnologia e Sociedade (CTS) articulase procurando explorar e compreender as maneiras com que a ciência moderna formata a cultura, os valores e as instituições modernas, e como os valores contemporâneos, por sua vez, formatam a ciência, incluindo nas discussões aspectos econômicos, políticos, éticos e ambientais da Ciência e Tecnologia (C\&T). Contextualizando a relação entre ciência, tecnologia e sociedade, este campo procura demonstrar que todo desenvolvimento científico-tecnológico é socialmente construído, e que existem sujeitos atuantes e interesses específicos no contexto do desenvolvimento científico e na construção de artefatos tecnológicos (BAZZO; Von LINSINGEN; PEREIRA, 2003).

As agendas de pesquisa, os financiamentos oferecidos pelos órgãos de fomento através de editais estabelecidos ou bolsas de pesquisa para esta ou aquela área, a internacionalização da pesquisa para estes ou aqueles países: são exemplos de tomadas de decisões estratégicas 
que fazem parte das políticas públicas de ciência e tecnologia. As razões das escolhas não são estritamente técnicas, não se trata de seleção natural: as escolhas são políticas e realizadas por "especialistas". Vivemos em um ambiente social onde a ciência teoricamente é mais livre em estruturas democráticas; no entanto, o que observamos na realidade é a presunção da demanda por aqueles que definem o que se pesquisa: criamse necessidades, não se parte delas. Diante deste controverso cenário, as perspectivas dos Estudos da Ciência, Tecnologia e Sociedade vem para contrapor a visão de ciência neutra, por defenderem que, na realidade, a sociedade influi no desenvolvimento da ciência e tecnologia, e a ciência e tecnologia contribuem

significativamente para desenvolvimento social (MANASSERO MAS; VÁZQUEZ, 1999).

Segundo López Cerezo (1998), a equação ciência + tecnologia $=$ riqueza + bem estar social reflete um modelo linear unidirecional de desenvolvimento (também chamado de modelo linear de inovação), no qual a pesquisa básica é necessariamente o ponto de partida, levando a aplicações tecnológicas, que, por sua vez, produziriam benefícios econômicos e sociais para toda a sociedade numa sequencia linear. Essa visão acompanha as políticas científica e tecnológica desde o final da Segunda Guerra Mundial (1945), principalmente entre os países do eixo norte. A "Big Science" incorporou a política econômica ao conhecimento científico e tecnológico e trouxe como aliado, financiador e gestor o Estado.

Para justificar os altos financiamentos públicos para pesquisa cientifica e tecnológica, leia-se armamentista, os Estados Unidos e a Europa passaram a difundir a ideologia de que o investimento em pesquisas científicas traria resultados suficientes para o desenvolvimento. Estabeleciase então uma nova forma de contrato social entre a comunidade cientifica e o estado (DAGNINO; THOMAS, 1999).

A crítica do Campo CTS à ciência pronta, importada dos centros para a periferia, se dá por acreditar que tal modelo, além de reforçar as relações de dependência históricocultural das decisões políticas, processo análogo às relações colonizadoras de outrora, contribuem para agravar disparidades regionais, fortalecendo as relações de dependências entre os países 
desenvolvidos e os que buscam desenvolvimento. Não se trata de ser um campo anti-tecnologia ou antiprogresso, como afirma Langdon Winner, e comungando com seu pensamento, "se a nossa linguagem moral e política para avaliar tecnologias incluir apenas categorias relativas a ferramentas e usos, se ela não incluir atenção ao significado dos projetos e arranjos de nossos artefatos, então ficaremos cegos" (WINNER, 1980, p. 125).

$\mathrm{Na} \quad$ América Latina, particularmente na Argentina em 1960, emergiu um movimento acadêmico que veio, com base nos pressupostos teóricos dos Estudos Sociais da Ciência e Tecnologia e da Teoria da Dependência, problematizar a hegemonia do modelo ofertista linear de ciência e tecnologia como proposta de desenvolvimento para a região. Com uma crítica anti-imperialista o movimento que ficou conhecido como Pensamento Latino-americano em Ciência Tecnologia e Sociedade (PLACTS) propunha naquele momento uma reflexão sobre as características do desenvolvimento da ciência e tecnologia, assim como seu papel no desenvolvimento econômico e social em regiões de contexto periférico no qual se encaixam os países latinoamericanos.

Os estudos do campo CTS produzidos naquele momento foram importantes para subsidiar as discussões e propostas que surgiriam com 0 intuito de criar uma independência tecnológica, um projeto nacional de ciência e tecnologia. Para Kreimer (2007) as produções intelectuais do PLACTS tinham a "preocupação política para encontrar formas $e$ instrumentos para o desenvolvimento de conhecimentos científicos e tecnológicos locais, para que pudesse ser adequado para as necessidades da região" (KREIMER, 2007, p.1).

O objetivo dessa geração era fazer com que a ciência e tecnologia virassem objeto de estudo público, como um tema ligado a uma estratégia de desenvolvimento econômico e social. Os pesquisadores do movimento, entre eles os argentinos Amilcar Herrera, Oscar Varsavski, Jorge Sábato, e os brasileiros José Leite Lopes, Mário Schenberg entre outros, ao persistirem na ideia de que a "periferia" precisava e tinha condições de desenvolver Ciência e Tecnologia com as características peculiares das necessidades dos países latino- 
americanos, fez emergir agências nacionais de ciência e tecnologia, políticas e uma série de estudos e discussões sobre políticas de ciência e tecnologia que viessem a mobilizar a C\&T como alavancas de desenvolvimento socioeconômico (KREIMER, 2007).

Sobre essa relação "centroperiferia" se faz necessário abrir um parêntese para, como ressalta Paty (1999), frisar a diferença entre "ciência periférica" e "ciência na periferia": a primeira seria marginal em termos de conteúdo de conhecimento e resultados, qualidade e quantidade, bem como no que diz respeito ao sistema científico, educacional e industrial. A segunda, nos permite admitir a possibilidade de que as principais contribuições para a ciência realmente se originam da chamada periferia, e que a formação científica pode chegar a níveis comparáveis ao alcançado nos países do "centro". Pesquisas notáveis são encontradas na periferia, alguns são tão bons como quaisquer estudos originários dos países de centro. O autor apresenta uma "longa lista de temas", incluindo a investigação microbiológica do brasileiro Oswaldo Cruz, para ressaltar sua opinião (PATY, 1999).
Apesar dos esforços do movimento PLACTS e da contribuição crítica à visão "etapista" da transferência de tecnologia, a década de 1980, particularmente no Brasil, simbolizou um passo atrás, um recuo para o campo. Enquanto celebrava-se a crescente institucionalização da ciência brasileira com o fortalecimento dos renomados órgãos como CAPES, CNPQ, SBPC e o Ministério da Ciência, Tecnologia e Inovação ao longo do século $X X$, e o processo de redemocratização não só do Brasil como de outros países latinos, no Campo CTS viu-se acirrado o embate filosófico com a comunidade cientifica (DIAS, 2010).

Visto como um movimento de visões utópicas, os pesquisadores de filosofia CTS se contrapõem ao discurso determinista da comunidade cientifica e de pesquisa, principalmente no campo das ciências básicas e engenharias, por estes apoiarem e ajudarem na difusão de um modelo de desenvolvimento cientifico e tecnológico apoiado na velha máxima da inovação tecnológica como via única para o desenvolvimento econômico. $\mathrm{O}$ que se materializou como política de C\&T, principalmente a partir da redemocratização política, foi um 
modelo de inovação tecnológica fomentada pela lógica neoliberal e da globalização onde caberia aos empresários fomentar a inovação no plano nacional.

Nesse contexto, as políticas científicas e tecnológicas refletem a ideia de vinculacionsimo da inovação tecnológica com os mercados econômicos, ou seja, o conhecimento tem de passar pela empresa privada para beneficiar a sociedade. As empresas privadas passam a ser fonte exclusiva de competitividade nacional. Já o Estado tende a manter as condições necessárias para o desenvolvimento de atividades de formação de pessoal e de Pesquisa e Desenvolvimento (P\&D) que atendam ao mercado, com a promoção de empresas de "alta tecnologia" e com escassa relevância para a vida da maioria da população (DAGNINO, 2013, p. 38).

Desde então prevalece nas PCTs a influência do modelo linear agora subsidiado pela Teoria da Inovação que, "baseada na concepção liberal da separação entre o mundo público e privado, supõe que o conhecimento produzido na sociedade só pode chegar a proporcionar a esta um resultado positivo caso seja usado pela empresa" (DAGNINO, 2010, p. 35). A concepção nacionalistadesenvolvimentista das décadas de 1960 e 1970 passa a ser substituída por uma visão que entende as empresas privadas como fonte exclusiva de competitividade nacional, pensamento que Dias (2010) chama de "Privatização da PCT".

Dando um passo a frente nas discussões sobre uma PCT alinhada com os estudos CTS, que além de defender a ruptura deste modelo de política de C\&T, conclama a participação de novos atores na elaboração de política de ciência, tecnologia e inovação, a Associação Brasileira de Estudos Sociais das Ciências e das Tecnologias (ESOCITE$\mathrm{BR}$ ), se posicionou a favor de uma outra PCT, após debates ocorridos no congresso da Associação, em Curitiba, no ano de 2013, onde mais de 500 pesquisadores debateram "Outro Desenvolvimento é Possível?”.

A "Carta de Curitiba, 2013", como ficou conhecido o documento, defende que um novo desenvolvimento é possível a partir da implementação e avaliação de novos tipos de políticas científicas e tecnológicas coerentes com o que a Associação pontua como "ideias-força": o desenvolvimento deve 
ser: (a) social e caracterizado pela busca por práticas sócio-técnicas inclusivas; (b) problematizado em suas muitas faces e contradições socioculturais; (c) ambientalmente sustentável e contraposto à insustentabilidade provocada pelo capital; (d) baseado em práticas científicas e tecnológicas solidárias e igualitárias enraizadas nos movimentos sociais (ESOCITE-BR, 2013).

Para avançar, a ESOCITE-BR (2013) recomenda que se discuta tanto na academia quanto na sociedade: agendas de pesquisa, transformação social e inovação, no sentido de tornálas mais aderentes ao processo de democratização em curso no país; o combate às visões hierarquizantes, autoritárias, preconceituosas de ciência e tecnologia, valorizando e interagindo com os conhecimentos plurais, produzidos por trabalhadores e

\section{O NORDESTE DA PCTI}

O PCTI foi pensado para qual o Nordeste? Os dados listados a seguir ilustram os desafios de se pensar um plano de desenvolvimento para a região. São indicadores que, dependendo do diagnóstico que se quer demonstrar ou da percepção dos investigadores, mostram realidades diferentes. Assim como aplicável na trabalhadoras, por comunidades tradicionalmente excluídas (indígenas, remanescentes de quilombolas) e a necessidade de reforçar, no âmbito das PCTI, o estímulo aos agentes interessados no novo estilo de produção, consumo e transformação e ao desenvolvimento de soluções tecnocientíficas adequadas (Tecnologia Social).

A Carta de Curitiba desenha pontos importantes para a discussão proposta por esse artigo, que é analisar o PCTI/NE a partir das premissas CTS para um Planejamento da Ciência e Tecnologia. Diante do que já se foi explicitado, entende-se que um Plano ou Política de C\&T, para atender aos princípios democráticos, deve prezar pela efetiva incorporação de um conjunto mais diversos de atores no seu processo de elaboração e debate.

"metáfora do copo meio cheio, meio vazio", para o pesquisador "otimista", que enxerga o copo meio cheio, a região obteve crescimento de renda maior que a média nacional com os investimentos dos últimos 10 anos. Para o pesquisador "pessimista", em dez anos a economia nordestina não 
conseguiu ampliar sua participação na economia nacional para mais de $1 \%$.

O Governo Federal, por meio dos Programas de Aceleração do Crescimento (PAC 1 e 2) contribuiu para uma leve modernização do setor industrial relacionado a infraestrutura. Nesse período, entre 2007 e 2011, foram criados termoelétricas, portos, estaleiros, refinarias, indústria automotiva, siderúrgicas, entre outros investimentos considerados importantes para a aceleração do crescimento regional. Os indicadores da primeira década dos anos 2000 mostram que a região não conseguiu aumentar sua participação no acumulado da economia nacional, que em 2010 correspondeu a 13,4\%, e no ano 2000 era de12,4\%.

Outros indicadores que devem ser levados em consideração quando se fala de desenvolvimento regional para o Nordeste são os relacionados à educação. Assim como nas demais regiões brasileiras, houve um aumento significativo na expansão do ensino superior a partir do Programa de Apoio a Planos de Reestruturação e Expansão das Universidades Federais (Reuni), que tem como principal objetivo ampliar o acesso à educação superior. Dados de 2011 listam que a região contava com 432 Instituições de Ensino Superior, sendo 63 destas públicas.

A oferta de cursos de Pósgraduação acompanhou a ascensão. Atualmente a região conta com 862 programas de pós-graduação, o que implica um aumento de mestres e doutores. Segundo o estudo do CCGE (2016), a formação de mestres cresceu $823,7 \%$ no Nordeste, passando de 1.116, no ano de 1996, para 9.193, em 2014. No caso do doutorado, o aumento foi de $5.980 \%$, passando de 40, em 1996, para 2.392, em 2014.

No entanto, em 2010, 54\% dos analfabetos brasileiro, com 15 anos ou mais, estavam no Nordeste. Neste mesmo período, $7,1 \%$ dos nordestinos com 25 anos ou mais tinham superior completo, segundo o Censo de 2010. Esse breve contexto socioeconômico mostra a complexidade da região, que apesar de ter conseguido inserir-se e manter-se ativo na economia nacional, não pode ignorar suas fragilidades socioeconômicas. 


\section{O PLANO DE CIÊNCIA, TECNOLOGIA E INOVAÇÃO PARA O DESENVOLVIMENTO SUSTENTÁVEL DO NORDESTE BRASILEIRO (PCTI/NE): ANÁLISE E DISCUSSÃO}

O desenvolvimento do PCTI/NE iniciouse em 2013, a partir da solicitação dos secretários estaduais de CT\&l e presidentes das Fundações de Amparo a Pesquisas à CGEE, sendo incluído no contrato de gestão mantido entre MCTI e o Centro. Assim, seu escopo foi construído coletivamente, com a participação das secretarias estaduais de CT\&l e das FAP dos onze Estados que compõem a área de influência (Alagoas, Bahia, Ceará, Maranhão, Paraíba, Pernambuco, Piauí, Rio
Grande do Norte, Sergipe, Espírito Santo e Minas Gerais - norte mineiro e bacia do Jequitinhonha-Mucuri), adotando um horizonte de 20 anos para as metas e ações propostas (CENTRO DE GESTÃO E ESTUDOS ESTRATÉGICOS, 2014). Procurando expressar as demandas dos gestores de CT\&l e governos locais, a elaboração do plano partiu da análise documental e perpassou as etapas apresentadas na figura 1 abaixo:

Figura 1 - Processo de elaboração do PCTI/NE

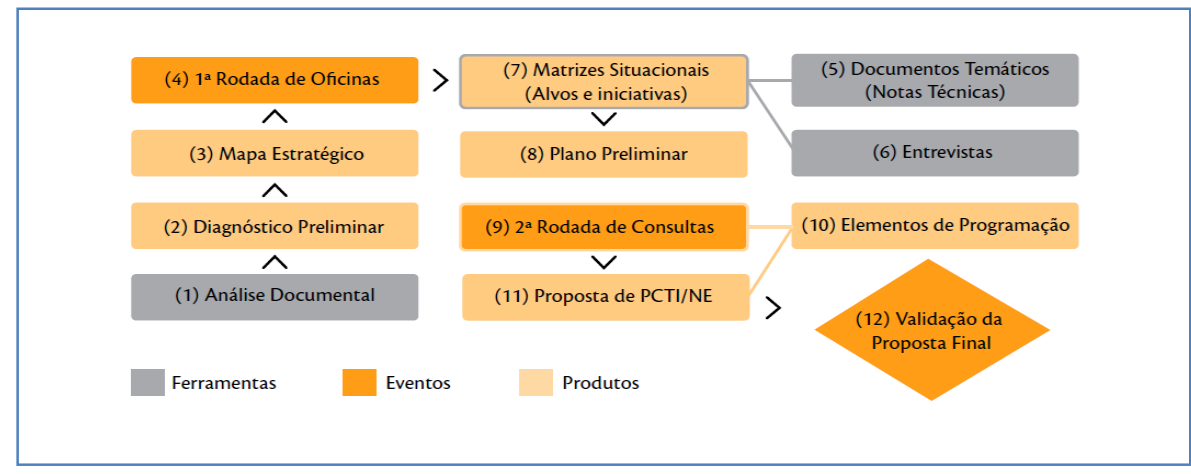

Fonte: Centro de Gestão e Estudos Estratégicos (2014).

A partir da análise documental sobre o desenvolvimento e o sistema de CT\&I do nordeste foi elaborado um diagnóstico preliminar, que culminou no desenvolvimento do Mapa Estratégico, em que foram apontados os principais desafios regionais em CT\&l, condensados em três categorias: promover a consolidação da base científica e tecnológica regional; estimular o empreendedorismo, a partir do incremento da competitividade e da adoção de práticas inovadoras e implantar nova agenda de CT\&l, voltada para o aproveitamento das potencialidades e oportunidades 
regionais (CENTRO DE GESTÃO E ESTUDOS ESTRATÉGICOS, 2014).

O diagnóstico e a elaboração do Mapa Estratégico inicial subsidiaram a $1^{\text {a }}$ rodada de consulta aos atores estaduais, protagonizada pelas Secretarias Estaduais de CT\&I e FAP dos Estados da região de influência. $A$ intenção desta primeira rodada foi identificar referenciais estratégicos e táticos para o Plano, sob a forma de "alvos estratégicos e iniciativas concretas" (CENTRO DE GESTÃO E ESTUDOS ESTRATÉGICOS, 2014, p.92).

Construídas de forma colaborativa, as propostas apontadas nas consultas foram estruturadas mobilizando-se especialistas e representantes dos governos federal, estaduais e municipais, trabalhadores e empresários, bem como de outros segmentos das comunidades interessadas, contando com cerca de 250 atores regionais.

No entanto, houve a mobilização majoritária de especialistas da realidade da CT\&l da região, considerados pelo documento "capazes, portanto, de emitir opiniões alinhadas com os propósitos das Oficinas" (CENTRO DE GESTÃO E ESTUDOS ESTRATÉGICOS, 2014, p.93). Paralelamente, foram encomendadas sete notas técnicas a especialistas em desenvolvimento regional e sistema regional de CT\&I, visando obter informações sobre 0 quadro atual e as perspectivas futuras para as questões socioeconômicas do Nordeste, analisar o sistema de CT\&I regional e sua conexão com o ambiente da CT\&l do País e discutir as oportunidades de desenvolvimento tecnológico e econômico abertas à economia nordestina (CENTRO DE GESTÃO E ESTUDOS ESTRATÉGICOS, 2014).

Outros especialistas ainda foram convidados a contribuir, por meio de entrevistas, para que se ampliasse o escopo e consistência dos resultados obtidos na $1^{\underline{a}}$ rodada de consulta. A partir daí elaboraram-se as Matrizes Situacionais, com apontamento dos alvos e iniciativas concretas, dando origem a uma versão preliminar do Plano, que foi levada à $2^{\underline{a}}$ rodada de consulta, com participação dos representantes das Secretarias Estaduais de CT\&I e FAP. Segundo o PCTI/NE:

As metas foram apontadas a partir da análise combinada dos estrangulamentos levantados no sistema regional de CT\&I com objetivos 
estratégicos. As iniciativas foram selecionadas dentre o conjunto de mais de 500 enunciados (propostas), que foram extraídos das dez rodadas de oficinas e dos estudos técnicos e entrevistas realizadas. As Iniciativas foram qualificadas e definidas a partir da interpretação desses conteúdos frente aos objetivos e desafios (CENTRO DE GESTÃO E ESTUDOS ESTRATÉGICOS, 2014, p.92).

O trabalho resultou em objetivo geral do plano (alinhado ao PCTI nacional), objetivos estratégicos, metas gerais e transversais, linhas de ação e diretrizes e iniciativas. A partir daí, objetivando a construção de um direcionamento concreto para a CT\&I do Nordeste, e considerando os desafios regionais delineados no Plano, cinco objetivos estratégicos foram delimitados: consolidar um sistema regional de CT\&I com capacidade endógena de formulação e implementação de políticas e apto a interagir local, nacional e globalmente, transformar o Nordeste em referência mundial na P\&D e inovação para a convivência com a seca, combate à desertificação e adaptação às mudanças climáticas, ter o Nordeste como referência na $P \& D$ e inovação para o desenvolvimento inclusivo, com destaque para a geração de conhecimentos que tenham foco no território, na biodiversidade e na cultura regionais, ampliar na região o conjunto de empresas competitivas, a partir de suas capacidades e competências em tecnologia e inovação, que apresentem desempenho comparável às melhores referências nacionais e globais e desenvolver na região uma base técnico-científica de excelência, globalmente conectada, capaz de produzir conhecimentos de ponta, de alto conteúdo científico e tecnológico (CENTRO DE GESTÃO E ESTUDOS ESTRATÉGICOS, 2014, p.94).

As metas refletem, numericamente, o caminho a ser percorrido para o alcance dos objetivos apontados no período de tempo determinado no Plano. Estão intrinsecamente ligadas ao aumento dos gastos em CT\&I na região, em nível federal, estadual e privado. As diretrizes e iniciativas foram sugeridas pelos atores regionais e estaduais participantes das oficinas e especialistas consultados.

Quatro linhas de ação foram estabelecidas para 0 Objetivo Estratégico 1, centradas no fortalecimento das instituições estaduais responsáveis pela CT\&I, 
incluindo a melhora de seus quadros gestores (recursos humanos) e ampliação de infraestrutura. Para o segundo Objetivo Estratégico, outras quatro linhas voltam-se para a produção, disseminação e cooperação (nacional e internacional) de conhecimentos relacionados à questão do semiárido. Para o terceiro, o foco está no desenvolvimento de redes de geração e difusão do conhecimento para a inovação inclusiva, baseada nos contextos locais e tecnologias sociais. Para o quarto objetivo, as linhas de ação centram-se principalmente na consolidação das instituições de pesquisa e inovação, sua interação com as empresas regionais e a atração de centros de pesquisa e desenvolvimento, voltados para as demandas de $P \& D$ regionais. Para 0 Objetivo Estratégico quinto, o foco está na formação de alto nível em CT\&l e internacionalização da ciência produzida na região. Para cada linha de ação, diretrizes e iniciativas concretas foram delineadas.

A previsão de recursos para a realização do Plano (advindos do Governo Federal, Estadual e empresariado), é abrangente para os objetivos 4 e 5 , e mais comedida para os objetivos 1,2 e 3 . O modelo de gestão sugerido para $0 \quad \mathrm{PCTI} / \mathrm{NE}$ baseia-se na ideia de Gestão e Governança Territorial, que implica no desenvolvimento de ações estratégicas em CT\&I ligadas à dimensão territorial, incluindo a proposta do Fórum Consecti/Confap-Nordeste para instância institucional e deliberativa do Plano (CENTRO DE GESTÃO E ESTUDOS ESTRATÉGICOS, 2014).

Encomendado pelas Secretarias Estaduais de CT\&I e FAPs, o desenvolvimento do PCTI/NE se dá baseado em uma visão instrumental da CT\&I, explicitado fortemente em seu objetivo mais geral: "O PCTI/NE tem por mote a transformação da CT\&I em instrumento para o desenvolvimento". Embutida nesta assertiva está a aceitação do chamado modelo linear de inovação, em que ciência e tecnologia são uma etapa, indiscutivelmente fundamental, para a riqueza, bem estar social e desenvolvimento. Este constitui o contexto de desenvolvimento de todo o documento e reforça a não proximidade com o que propõe os Estudos Sociais da Ciência e Tecnologia.

O Plano dialoga com as complexidades e desafios do Nordeste quando leva em consideração a necessidade de fortalecer na região 
estratégias de P\&D e inovação para a convivência com a seca, combate à desertificação e adaptação às mudanças climáticas. No entanto, é notória a concepção: a) eficientista, uma vez que prega competitividade, competência e desempenho tecnológico comparável as melhores referências globais em tecnologia e inovação; b) determinista, já que qualifica a CT\&I como instrumento para o desenvolvimento regional; e c) centralizadora, em razão de suas ações partirem da "voz" dos "especialistas".

A elaboração do plano, ainda que se apresente como colaborativa ou participativa, privilegia em seu âmbito a participação de agentes governamentais (federais, estaduais, municipais) e especialistas em CT\&I, aos quais foram solicitadas notas técnicas e entrevistas, em busca do que se chamou de "informações estruturadas" e alinhadas com os propósitos das oficinas, ou seja, serviram como um "ritual", um trâmite do processo para balizar as estratégias que se alinhavam de forma "qualificada" com o objetivo fundamental da proposta. Não foram usadas como construção e sim como ferramenta de confirmação de objetivos. Cenário que não condiz com o que se espera de um plano construído de maneira colaborativa, participativa, democrática.

Observa-se, portanto, que desde sua gênese o PCTI/NE se desenvolve de maneira enviesada, desprivilegiando uma gama de atores sociais que poderia contribuir para que 0 documento refletisse outros olhares, diretrizes e iniciativas. Outro ponto passível de analise é a ênfase dos objetivos estratégicos dada a questões relacionadas à formação de pesquisadores e recursos humanos qualificados, assim como visto na critica do PLACTS nos anos 60 , repetese o cenário "tripla hélice", onde o estado é o formador de mão de obra qualificada para que 0 mercado absorva essa mão de obra e promova o desenvolvimento regional esperado pela sociedade.

\section{CONSIDERAÇÕES FINAIS}

O PCTI/NE é uma proposta transformar o Nordeste em referência encomendada a especialistas para mundial de P\&DI, dando 
competitividade às empresas para que se alcance os patamares nacionais e globais de competência tecnológica. $\mathrm{O}$ foco está na edificação de uma base técnico-científica para produção de conhecimento de alto conteúdo científico e tecnológico. É um plano pensado dentro da lógica capitalista do neoliberalismo global e para atender suas demandas, onde a eficiência e competitividade são características fundamentais.

Em planos como o PCTI, analisado por esse paper, as demandas são mercadológicas, sendo suas estratégias pensadas para atender necessidades do mercado detentor do capital e não de uma agenda da sociedade. Não há espaço nesses modelos de planejamento para conhecimentos plurais e outros estilos de produção e consumo, principalmente quando o que se visa projetar é o desenvolvimento cientifico e tecnológico, pois não há espaço para Tecnologia Social na lógica do estado neoliberal.

Sendo assim, uma nova agenda de pesquisa que tenha força para romper com a lógica de orientação da construção da Política de C\&T é essencial para a melhoria das condições de vida do conjunto da sociedade. Ou há uma mudança de paradigma ou as políticas continuarão a reproduzir e aprimorar o modelo em curso, onde a agenda de pesquisa não é determinada pelas demandas da sociedade e sim pelas agências de pesquisa que as financiam.

\section{REFERÊNCIAS}

BAZZO, W. A.; Von LINSINGEN, I.; PEREIRA, L. T. do V. Introdução aos estudos CTS (Ciência, Tecnologia e Sociedade). Madrid: OEI, 2003.

BRASIL. Ministério da Ciência, Tecnologia e Inovação. Estratégia Nacional de Ciência, Tecnologia e Inovação 2016-2019. Brasília: MCTI, 2016.

CENTRO DE GESTÃo E ESTUdOS ESTRATÉGICOS (CGEE). Plano de Ciência, Tecnologia e Inovação para o desenvolvimento sustentável do Nordeste Brasileiro. Brasília, 2014. em: 
<http://www.cgee.org.br/publicacoes/documentos_tecnicos.php>. Acesso em: 19 ago. 2016.

DAGNINO, R.; THOMAS, H. La Política Científica y Tecnológica en América Latina: nuevos escenarios y el papel de la comunidad de investigación. Revista REDES, v. VI, n. 13, mayo de 1999, p. 49-74. Disponível em: <http://www.unq.edu.ar/advf/documentos/51d5b79c6fe6b.pdf > Acesso em: 19 ago. 2017.

DAGNINO, R. (Org.). Estudos sociais da ciência e tecnologia e política de ciência e tecnologia: abordagens alternativas para uma nova América Latina. Campina Grande: EDUEPB, 2010.

DAGNINO, R. O Pensamento Latino-Americano em Ciência, Tecnologia e Sociedade (PLACTS) e a obra de Andrew Feenberg. In: Ricardo T. Neder (org.). A teoria crítica de Andrew Feenberg: racionalização democrática, poder e tecnologia. Brasília: Observatório do Movimento pela Tecnologia Social na América Latina / CDS / UnB / Capes, 2013.

DIAS, R. de B. A agenda da política científica e tecnológica brasileira: uma perspectiva histórica. In: DAGNINO, R. (Org.). Estudos sociais da ciência e tecnologia e política de ciência e tecnologia: abordagens alternativas para uma nova América Latina. Campina Grande: EDUEPB, 2010.

ESOCITE-BR (2013), Por uma Democratização da Ciência e Tecnologia. Disponível em: <http://www.esocite.org.br/carta-de-curitiba-por-uma-democratizacaoda-ciencia-e-da-tecnologia/>. Acesso em: 02 set. 2016.

KREIMER, P. Social Studies of Science and Technology in Latin America: a field in the process of consolidation. Science, Technology \& Society, n.12, v.1, p. 1-9, março de 2007.

Disponível

em:

http://journals.sagepub.com/doi/pdf/10.1177/097172180601200101. Acesso em: 02 set. 2016. 
LÓPEZ CEREZO, J. A. Ciencia, Tecnologia y Sociedad: el estado de la cuéstión en Europa y Estados Unidos. Revista Iberoamericana de Educación, OEI, n. 18, set./dez. 1998. Disponível em: http://www.oei.es/oeivirt/rie18a02.htm. Acesso em: 30 jul. 2016.

MANASSERO MAS, M. A.; VÁZQUEZ, A. A. Actitudes hacia la influencia de la sociedad en la ciencia y la tecnología. Arbor, v.162, n.637, 45-72. Disponível em: http://arbor.revistas.csic.es/index.php/arbor/article/view/1681/1796. Acesso em: 02 set. 2016.

PATY, M. Comparative history of modern science and the context of dependency. Science Technology Society, 1999, v. 4, n. 2, p. 171-204. Disponível em: http://journals.sagepub.com/doi/pdf/10.1177/097172189900400203. Acesso em: 02 set. 2016.

SOUSA, C. M. Relativizando ciência e comunicação. Brasília: Empresa Brasil de Comunicação, $2004 . \quad$ Disponível em: http://memoria.ebc.com.br/agenciabrasi//noticia/2004-01-23/relativizando-ciencia-ecomunicacao. Acesso em: 26 jul. 2016.

WINNER, L. Do Artifacts have Politics? Daedalus, v. 109, n. 1, p. 121-136 Disponível em: <https://transitiontech.ca/pdf/Winner-Do-Artifacts-Have-Politics-1980.pdf > Acesso em: 19 ago. 2017.

Recebido em: 04/09/2017

Aprovado em: 24/09/2017 